SciBX

\section{BETting on Th17 cells}

\section{By Lev Osherovich, Senior Writer}

Researchers at Constellation Pharmaceuticals Inc. have established a connection between BET bromodomain proteins and pathogenic T helper type 17 cells. ${ }^{1}$ The findings hint at therapeutic opportunities for this epigenetic target class in autoimmunity and inflammatory disease, but further details about tolerability and specificity of current BET inhibitors are needed.

BET bromodomains are protein modules that bind to acetylated lysine residues of histones, the principal components of chromatin. Small molecule BET inhibitors mimic acetylated lysine and prevent BET proteins from recognizing chromatin, leading to transcriptional changes that arrest cell proliferation and differentiation.

Most efforts to target BET proteins have focused on cancer. BET inhibitors from Constellation, GlaxoSmithKline plc and Oncoethix S.A. are in Phase I testing for a range of tumor types.

In 2010, an international team that included The Rockefeller University and GSK reported that GSK525762, a broad-spectrum BET inhibitor in Phase I testing for epithelial cancer, also had potent anti-inflammatory effects in macrophages. ${ }^{2}$ Rab Prinjha, VP and head of the EpiNova epigenetics discovery performance unit at GSK, said that results of the trial are expected in 2014.

Now, a Constellation team has shown that BET proteins influence the development and activation of T helper type 17 (Th17) cells, which are dysregulated in autoimmune diseases including multiple sclerosis (MS) and rheumatoid arthritis (RA).

"There was already a good notion that this family of proteins controls inflammation," said Jose Lora, team leader and executive director of preclinical sciences at Constellation. "The novelty of our recent paper is that these BET proteins control an important differentiation pathway in T cells."

Lora previously was director and head of immuno-epigenetics biology at GSK.

He said that the findings suggest BET proteins regulate inflammation by controlling expression of Th17 cell-specific proinflammatory cytokines.

"We are showing that this pathway is a fundamental mediator of transcription of these cytokines and show in animal models that this is a reasonable entry point for therapeutics," he added.

Lora's team treated undifferentiated human T cells with JQ1, a broadspectrum, small molecule BET inhibitor identified by researchers at Harvard Medical School, ${ }^{3}$ and then subjected the cells to conditions that promoted differentiation of various helper cell subtypes.

JQ1 prevented differentiation of Th17 cells but not of Th1, Th2 or $\mathrm{T}_{\text {reg }}$ cells, whereas vehicle had no effect.

In naïve $T$ cells that had not yet developed Th17 cell characteristics, JQ1 blocked the expression of Th17 cell-associated cytokines including IL-17, IL-21 and granulocyte macrophage colony-stimulating factor (GM-CSF; CSF2).

The team obtained a similar result in actual Th17 cells. That finding suggests that BET blockade not only could block the initiation of the Th17 cell response but also potentially could shut down active Th17 cells.

In mouse models of MS and RA, JQ1 decreased Il-17 production and disease severity compared with vehicle.

Results were reported in The Journal of Experimental Medicine.

\section{Helping the helpers}

Although the findings provide proof of concept for therapeutic intervention with BET inhibitors in Th17 cell-driven autoimmune diseases, the precise role of BET proteins in autoimmunity is still up for debate.

For example, the new findings contrast with last year's report in the Proceedings of the National Academy of Science from a team led by Anjana Rao that found that GSK525762 prevented the initiation of the Th1 cell response but could not halt ongoing autoimmune activity. ${ }^{4}$ Roo is a professor of signaling and gene expression research at the La Jolla Institute for Allergy \& Immunology.

In that study, GSK525762 blocked "the initial phases of $\mathrm{T}$ cell differentiation with specific and selective effects driven in a context-specific manner by signature cytokines more in Th1 than Th17 cells," said Prinjha, who was a coauthor of Rao's study.

Prinjha thinks that methodological differences between the two experiments could account for the divergent conclusions. He said that Rao's team used adoptive T cell transfer for its in vivo work, whereas Lora's team did much of its work in vitro in human T cells and in mouse disease models with relatively unmanipulated immune systems.

Alexander Tarakhovsky, a professor in the laboratory of immune cell epigenetics and signaling at Rockefeller University, said that the new findings underscore the limited ability of animal models to capture the full complexity of autoimmune disease.

"Current models of autoimmunity typically focus on a particular type of immune response, but such studies are not sufficiently inclusive of other aspects of immunity," he said.

Thus, said Tarakhovsky, the two teams' conclusions "are not mutually exclusive and could result from different experimental setups." 


\section{ANALYSIS}

\section{TARGETS \& MECHANISMS}

Tarakhovsky led the team that first reported the anti-inflammatory effects of BET inhibitors. ${ }^{2} \mathrm{He}$ is collaborating with GSK to characterize the immunological effects of the company's BET compounds.

Lora concurred that "the difference in models probably explains the differences" between the two studies.

Nevertheless, it is not certain that blocking Th17 cell activity is the main effect of BET inhibitors on the immune system.

"It is still unclear what is the most important site of action of the BET inhibitors," said Prinjha. "Our adoptive transfer experiments in the Rao paper went some way to showing the importance of Th1 cells, but more work is needed to characterize other immune cell types."

Hozefa Bandukwala, first author on last year's PNAS paper and now a principal scientist at Pfizer Inc., said that BET inhibitors likely affect a broad range of immune cells besides $\mathrm{T}$ helper cells. company's lead product in the space, CPI-0610, is in Phase I testing for lymphoma. Constellation did not disclose details about that compound's selectivity.

Meanwhile, genetic methods could help determine which BET proteins are working in which immune cells to drive autoimmunity.

"To get an answer about which specific proteins and cells are involved in which aspects of autoimmune disease, we would need conditional genetic knockouts," said Bandukwala.

Lora and Prinjha said that ongoing Phase I trials of pan-BET inhibitors in oncology should lead to insights about the immunological effects of blocking this protein family.

"The ongoing trials in oncology are going to be very informative. This is the first time that we'll see the safety profile of these compounds and how well they're tolerated," said Lora.

"I suspect the final choices of immuno-

\section{Red light}

Tarakhovsky said that uncertainty about what exactly BET inhibitors do to immune function should serve as a warning against leaping into the clinic.

He said that BET proteins appear to affect the development and activity of multiple immune cells, and thus he thinks that current BET inhibitors are likely to have broad immunosuppressive effects.

Current BET inhibitors are relatively unselective and are likely to hit multiple members of the BET protein family.

"In fact, we know very little about the true spectrum of the effects of these compounds," he said.

Improving the selectivity of BET inhibitors is a logical way to help position the molecules in autoimmune diseases.

Humans have at least nine BET proteins, the best characterized of which include bromodomain containing 2 (BRD2) and BRD4. Many other proteins feature bromodomains that are potentially affected by BET inhibitors.

"One potential area for improving the safety profile of BET inhibitors is to narrow the specificity to BRD2 and BRD4, but because all bromodomains are very similar, it will be challenging to achieve selectivity for one over the other," said Lora.

Lora added that Constellation has a discovery-stage program to identify selective BET inhibitors for autoimmune and inflammatory disease. The inflammatory disease indication will be driven by data that emerge from our ongoing clinical studies rather than more preclinical work," said Prinjha.

Constellation did not disclose whether it has filed patents in connection with the JEM study.

\section{Osherovich, L. SciBX 6(44); doi:10.1038/scibx.2013.1246 \\ Published online Nov. 14, 2013}

\section{REFERENCES} doi:10.1084/jem.20130376

Contact: Jose M. Lora, Constellation Pharmaceuticals Inc.,

e-mail: jose.lora@constellationpharma.com

2. Nicodeme, E. et al. Nature 468, 1119-1123 (2010)

3. Filippakopoulos, P. et al. Nature 468, 1067-1073 (2010)

4. Bandukwala, H.S. et al. Proc. Natl. Acad. Sci. USA 109, 1453214537 (2012)

\section{COMPANIES AND INSTITUTIONS MENTIONED}

Constellation Pharmaceuticals Inc., Cambridge, Mass. GlaxoSmithKline plc (LSE:GSK; NYSE:GSK), London, U.K. Harvard Medical School, Boston, Mass. La Jolla Institute for Allergy \& Immunology, La Jolla, Calif. Oncoethix S.A., Lausanne, Switzerland Pfizer Inc. (NYSE:PFE), New York, N.Y. The Rockefeller University, New York, N.Y.
1. Mele, D.A. et al. J. Exp. Med.; published online Oct. 7, 2013; Cambridge, Mass. 